\title{
Deriving optical properties in the near infrared using an inverse Monte Carlo program
}

Karl A. Pope, Lihong V. Wang

Karl A. Pope, Lihong V. Wang, "Deriving optical properties in the near infrared using an inverse Monte Carlo program," Proc. SPIE 3914, Laser-Tissue Interaction XI: Photochemical, Photothermal, and Photomechanical, (13 June 2000); doi: 10.1117/12.388055

Event: BiOS 2000 The International Symposium on Biomedical Optics, 2000, San Jose, CA, United States 


\title{
Deriving Optical Properties in the Near Infrared using an Inverse Monte Carlo Program
}

\author{
Karl Pope ${ }^{1}$, Lihong Wang ${ }^{2}$ \\ 1. Candela Corporation, 530 Boston Post Road, Wayland, MA 01778 \\ 2. Texas A\&M University, 3120 TAMU, Collage Station, TX 77846-3120
}

\begin{abstract}
:
An Inverse Monte Carlo program was developed based on a scaleable Monte Carlo algorithm. This program determines the skin optical $\left(\mu_{\mathrm{a}}\right.$ and $\left.\mu_{\mathrm{s}}\right)$ properties in vivo using reflectance and thermal measurements as inputs from different skin types, very light to very dark. Some basic assumptions are made: 1) epidermal thickness is close to $100 \mu \mathrm{m}, 2$ ) the scattering in the epidermis is the same or similar to the dermal scattering, 3) the dermal absorption and scattering coefficients are similar between individuals. Experimental measurements of reflectance and temperature were taken. These were input into a pair of Inverse Monte Carlo programs that generated the optical properties for the different skin types. A single layer Inverse Monte Carlo model was employed to determine the optical properties of the dermis. A 2-layer Inverse Monte Carlo program was used to determine the epidermal optical properties.
\end{abstract}

KEYWORDS: Inverse Monte Carlo, Optical Properties, Near Infrared, In vivo

\section{INTRODUCTION}

Monte Carlo (MC) modeling was adapted from field of physic's studies of $\mathrm{x}$-ray scattering ${ }^{1-3}$ for biomedical applications in order to describe the distribution of light in tissue ${ }^{4}$. To apply Monte Carlo modeling to light-tissue interactions there is a growing need to better define the optical properties of tissue in vivo. While the sophistication of MC models increased to include, for example, multi-layers ${ }^{5}$ and edge effects ${ }^{6}$, the need for accurate absorption and scattering coefficients, $\mu_{\mathrm{a}}$ and $\mu_{\mathrm{s}}$ for varying skin types, has only increased.

Many different methods have been used to collect and analyze optical properties and have been described in great detail in other papers 7,8 . With few exceptions ${ }^{9,10}$ most of this work has been done ex vivo. Once tissue is removed from the body it begins to undergo changes such as dehydration, cell death, changes in blood content and transformation of state of $\mathrm{Hb}$. The time between preparation and measurements as well as the thickness of the sample will affect the outcome to some extent. In addition, there has been little work done looking at different skin types, light skin vs dark skin.

The problem that motivated this work was that optical properties from different sources ${ }^{8,11}$ \{http://omlc.ori.edu/news/ and http://www.medhpys.ucl.ac.uk/research/borg\} had significantly different values. When optical properties from these sources were input into a Standard MC (SMC) program and followed by a finite element model of thermal diffusion the output of reflectance and temperature rise did not correspond to what was measured in our lab. Since our development work is sensitive to the effects of the amount of melanin in skin, very white to very dark skin (Fitzpatrick Skin Types I to VI), the ability to construct MC models that take skin type into account is of great interest.

The goal of this paper is to describe a method of obtaining optical properties in vivo on different skin types from 2 easily obtained measurements; total reflectance and temperature rise.

\section{METHOD}

Figure 1 is a flow chart of the procedure that was carried out. Two types of data were collected in vivo, total reflection and the peak temperature after a laser pulse. Reflection measurements were taken using an 
integrating sphere setup as shown in Figure 2. The laser, a Candela GentleLASE PLUS ${ }^{\mathrm{TM}}$ (Wayland, MA) with a wavelength of $755 \mathrm{~nm}$ and a $3 \mathrm{~ms}$ pulse duration was used in these experiments. A high-speed silicon photo-detector (Det210, ThorLabs Newton NJ) was used to collect the output. The voltage was normalized using Spectralon (Lapsphere, North Sutton, NH) reflectance standards, $99 \%$ and $75 \%$, and calibrated to energies using a power meter. Variations in laser energy were normalized using a beam splitter and an energy meter (Ophir Nova meter and Ophir detector FL250, Danvers, MA). Reflectance data was collected from the inner forearm of each volunteer. Skin types varied from very white to very black, Fitzpatrick Skin Type I to VI.

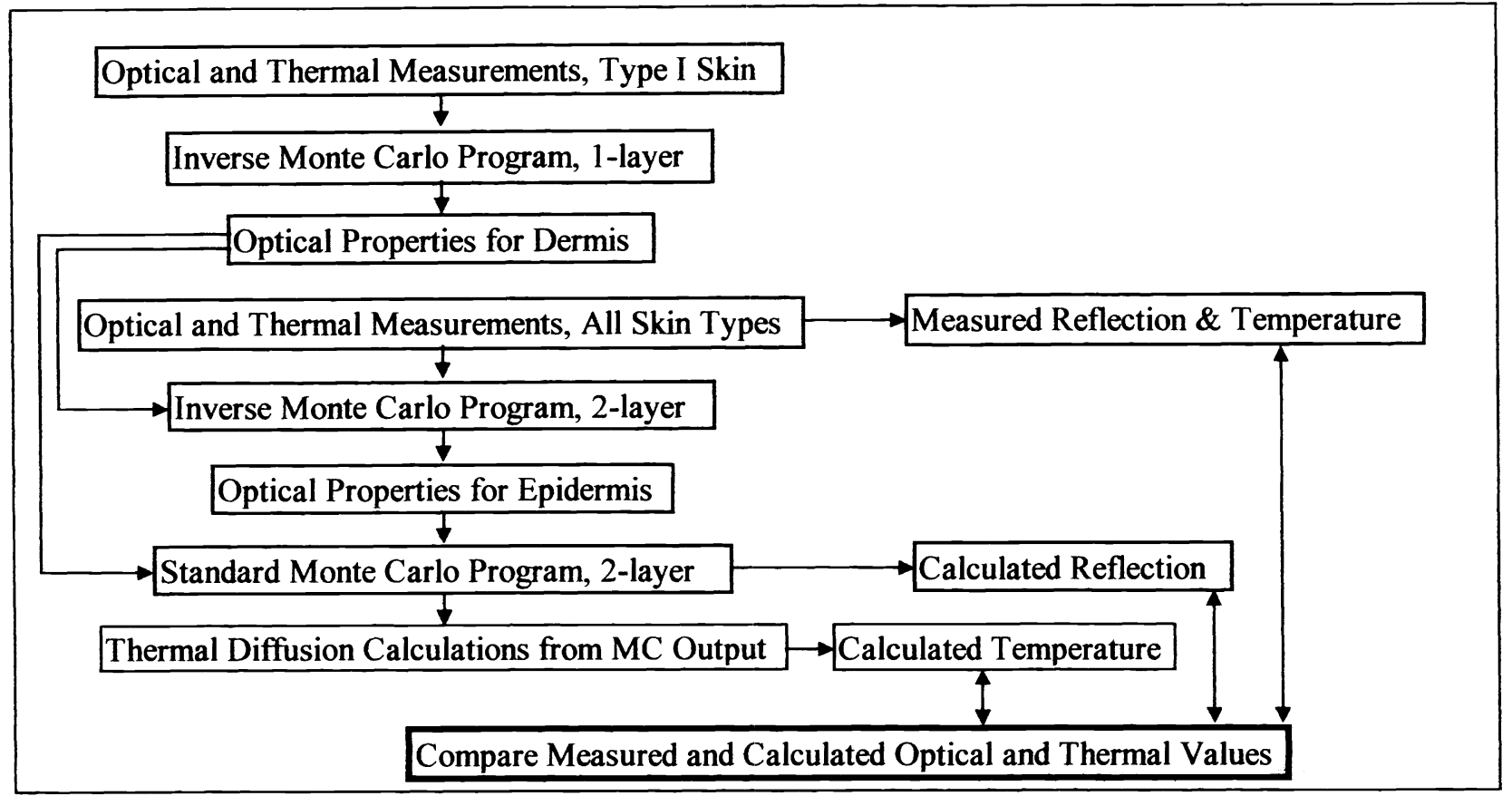

Figure 1: Flow chart of data collection, generation, and verification.

Skin temperature data were collected using a thermal camera, (Infametrics, Model 600, Bedford MA). Infametrics software was used to acquire temperature data on a single pixel at video frame rates $(60 \mathrm{~Hz})$. The GentleLASE PLUS laser was used at low fluence levels, 3 to $10 \mathrm{~J} / \mathrm{cm}^{2}, 3 \mathrm{~ms}$ laser pulse, to heat the skin without collateral damage. A beam splitter was used to sample the laser output power and correct for pulse to pulse variations. Three thermal measurements were taken at different spots on the inner arm of each volunteer and averaged.

Two Inverse Monte Carlo (IMC) programs, a 1- and 2-layer model, were employed to determine the optical properties from the reflection and temperature measurements. The 1- layer IMC model assumes that the reflection and temperature inputs are from a very white skinned individual, Fitzpatrick Skin Type I, and its' output are assumed to be the dermal optical properties. In the near IR the absorption in skin is dominated by melanin and to a lesser extent, blood. In Type I skin there is very little melanin present and the majority of the absorption is likely to occur in the dermis. So for very white skin the absorption coefficient $\left(\mu_{\mathrm{a}}\right)$ is dictated by the dermis. The epidermis is approximately $90 \pm 30 \mu \mathrm{m}$ and at wavelengths in the near infrared region there are not significant numbers of scattering events. It is therefore assumed that scattering in skin is dominated by the dermis. This is thought to be true for all skin types. The anisotropy was assumed to be 0.876 in these calculations

Diffuse reflection from a Monte Carlo program is defined by the ratio of $\mu_{\mathrm{s}} / \mu_{\mathrm{a}}$. The 1-layer IMC program first uses the measured diffuse reflectance from Type I skin, to iteratively calculate the ratio of $\mu_{\mathrm{s}} / \mu_{\mathrm{a}}$. Next, The 1layer IMC program uses the measured peak temperature from Type I skin to determine a unique $\mu_{\mathrm{a}}$ (derm). The laser pulse duration at $3 \mathrm{~ms}$ was determined to be sufficiently short that the system was considered to be thermally 
confined. Finally, after $\mu_{\mathrm{a}}(\mathrm{derm})$ is known then $\mu_{\mathrm{S}}$ (derm) can be calculated from the previously determined ratio of $\mu_{\mathrm{s}} / \mu_{\mathrm{a}}$.

Assuming that dermal properties do not change significantly between people, the optical properties obtained from the 1-layer IMC model can then be input as the dermal properties in the 2-layer IMC model for darker skin types. The 2-layer IMC program was next used to determine the epidermal absorption coefficient, $\mu_{\mathrm{a}}$ (epi) using the assumption that scattering is dominated by the dermis, the epidermal scattering coefficient was set to be the same as those of the dermis $\left(\mu_{\mathrm{s}}(\right.$ derm $)=\mu_{\mathrm{s}}(\mathrm{epi})$ ).

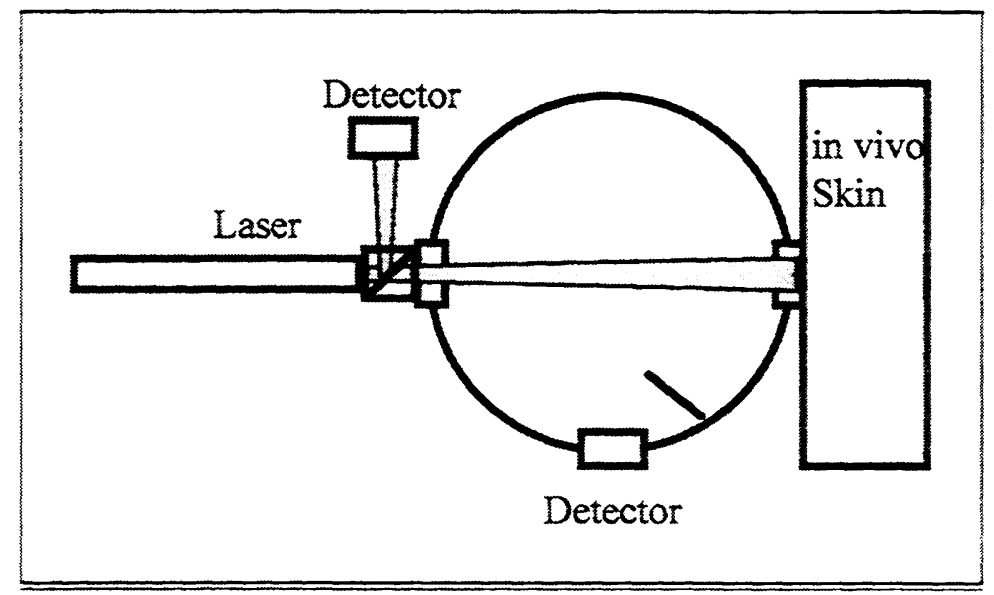

Figure 2: The collection geometry for reflection measurements using a laser and integrating sphere

The 2-layer IMC program collects only the calculated diffuse reflection data from the run. The 2-layer IMC program utilizes the measured diffuse reflection and adjusts the $\mu_{\mathrm{a}}$ (epi) that was input to the program until the calculated diffuse reflection matches the measured value.

\section{RESULTS}

The measured reflectance from very white skin, Fitzpatrick Skin Type I, was $59.2 \%$. After a $10 \mathrm{~J} / \mathrm{cm}^{2}, 3$ ms laser pulse at $755 \mathrm{~nm}$, the maximum temperature of $35.5^{\circ} \mathrm{C}$ was measured using a 8 to $12 \mu \mathrm{m}$ thermal camera. Using the 1-layer IMC model the values of $\mu_{\mathrm{a}}(\mathrm{derm})=0.410 / \mathrm{cm}$ and $\mu_{\mathrm{s}}(\mathrm{derm})=231 / \mathrm{cm}$ were calculated. Inputting these estimates into the 2-layer IMC for the optical properties of the dermal layer, an epidermal $\mu_{a}$ (epi) $=0.405 / \mathrm{cm}$ was calculated.

To validate the program, these optical properties from the Skin Type I were used in a Standard Monte Carlo (SMC) program. The calculated reflection was $58.8 \%$, which compares favorably with measured value of $59.2 \%$. When a thermal diffusion model was applied to the output of the Monte Carlo the skin temperature is calculated to reach $35.3^{\circ} \mathrm{C}$, again close to the measured $35.5^{\circ} \mathrm{C}$. This is a circular test, with the values of the epidermis and the dermis set to the same value, thus simulating the 1-layer model. The output is then used in a SMC and thermal diffusion model and the reflection and temperature results should match the starting values. This process validated the general concept and programming for skin Type I.

Testing a medium skin color, Fitzpatrick Skin Type III, the measured reflectance was $49.5 \%$. After a 10 $\mathrm{J} / \mathrm{cm}^{2}, 3 \mathrm{~ms}$ laser pulse at $755 \mathrm{~nm}$, the maximum temperature was measured at $49.3^{\circ} \mathrm{C}$. Using the same optical properties for the dermal layer as those derived from the Skin Type I, an epidermal $\mu_{\mathrm{a}}$ (epi) $=3.82 / \mathrm{cm}$ was calculated from the 2-layer IMC program. The Type III optical properties were then put into a SMC program. The calculated reflection was $51.3 \%$, which compares favorably with the measured value of $49.5 \%$. When a thermal diffusion model is applied to the output of the Monte Carlo the skin temperature is calculated to reach $74.8^{\circ} \mathrm{C}$, which is not close to the measured $49.3^{\circ} \mathrm{C}$. 
Finally when testing very dark skin, Fitzpatrick Skin Type VI, the measured reflectance was $27.9 \%$. After a $3 \mathrm{~J} / \mathrm{cm}^{2}, 3 \mathrm{~ms}$ laser pulse at $755 \mathrm{~nm}$, the maximum temperature of $52.6^{\circ} \mathrm{C}$ was measured. Using the same optical properties for the dermal layer as those derived from the Skin Type I, an epidermal $\mu_{a}=19.0 / \mathrm{cm}$ was calculated from the 2-layer IMC model. The optical properties derived from the Type VI skin were put into a SMC program and the calculated reflection was $31.9 \%$ compared to the measured value of $49.5 \%$. When a thermal diffusion model is applied to the output of the Monte Carlo the skin temperature is calculated to reach $70.7^{\circ} \mathrm{C}$, which is not close to the measured $52.6^{\circ} \mathrm{C}$. It should be noted here that only $3 \mathrm{~J} / \mathrm{cm}^{2}$ was used in the thermal measurements on the Type VI whereas $10 \mathrm{~J} / \mathrm{cm}^{2}$ was used for the Type I and III. In the thermal model temperature increase is proportional to the fluence. For Type VI skin in this simulation, a $10 \mathrm{~J} / \mathrm{cm}^{2}$ laser pulse would have resulted in a final measured and calculated temperature of $75^{\circ} \mathrm{C}$ and $137^{\circ} \mathrm{C}$ respectively.

\section{Discussion}

Abstracting optical properties from ex vivo skin has been studied extensively, but using optical and thermal measurements from in vivo skin provides a new and unique approach. This method has the advantage that the measurements are easy to obtain and there are few complicating factors that must be addressed to achieve optical properties.

The results from the 1-layer IMC program on very light skin showed a highly scattering tissue with low absorption, $\mu_{\mathrm{S}}=231 / \mathrm{cm}$ and $\mu_{\mathrm{a}}=0.410 / \mathrm{cm}$ respectively. When these numbers were used in a SMC program and followed by a thermal diffusion model, the reflection and final temperature results were closely related to the experimental measurements. This validated the programming of the IMC algorithm for skin Type I.

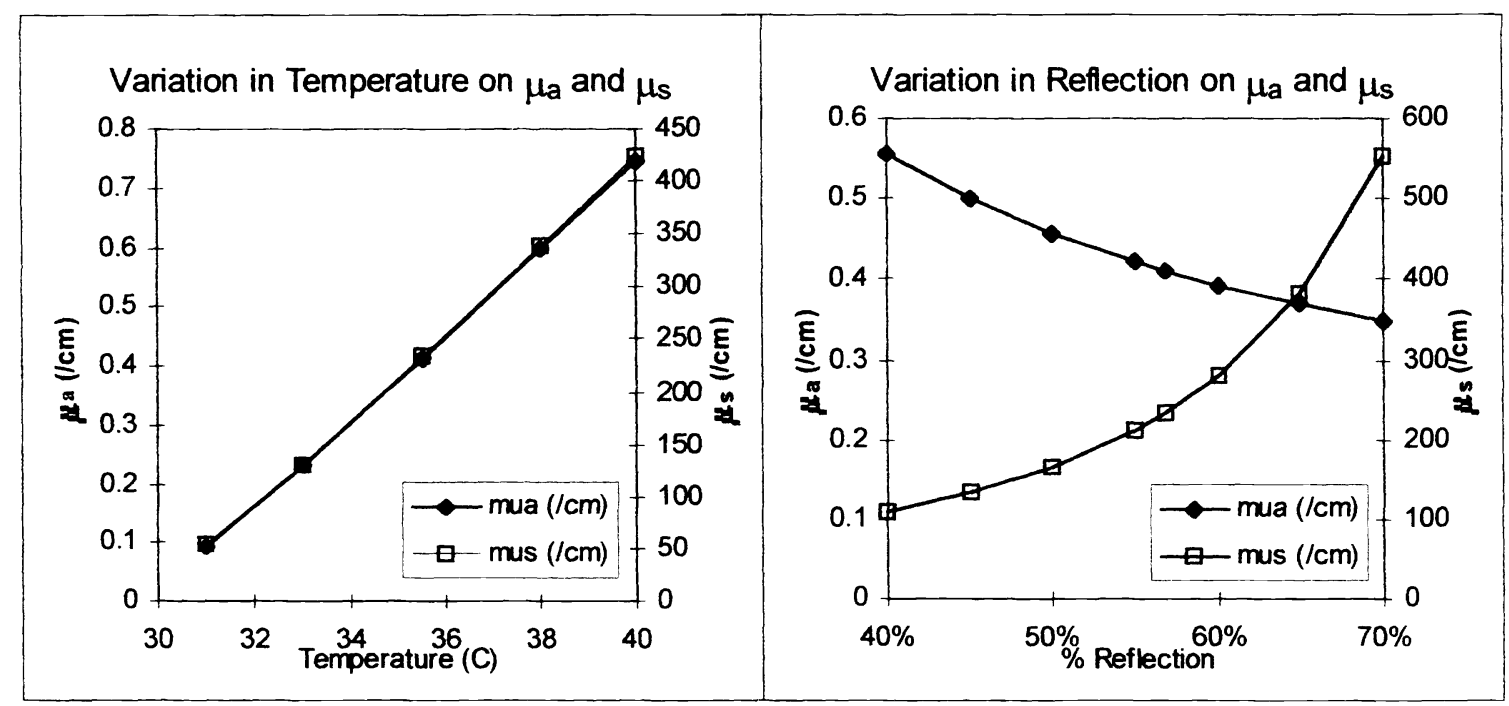

Figure 3 \& Figure 4: The effect on changes of temperature and percent reflection on $\mu_{\mathrm{s}}$ and $\mu_{\mathrm{a}}$ is shown. Increases in temperature measurements cause the calculated $\mu_{\mathrm{s}}$ and $\mu_{\mathrm{a}}$ to increase. Increases in percent reflection cause $\mu_{\mathrm{s}}$ to increase but $\mu_{\mathrm{a}}$ to decrease.

The Results from the 2-layer IMC model when employed on darker skin types was not as encouraging. When the resulting output optical properties from the 2-layer IMC program were run through the standard Monte Carlo model and thermal diffusion model, the reflection and final temperature were overestimated for both the medium and very dark skin types. However, the calculated reflection values were close to the measured ones, which was expected since the measured reflectance was used as an input to the IMC program.

To understand the source of the thermal error, the effect of under or over estimating the measured values for either the total reflection or final temperature, were considered. For changes in the final peak temperature, Figure $3, \mu_{\mathrm{s}}$ and $\mu_{\mathrm{a}}$ move together, as the final temperature increases so does the $\mu_{\mathrm{s}}$ and $\mu_{\mathrm{a}}$. For variations in the 
reflection, Figure $3, \mu_{\mathrm{S}}$ and $\mu_{\mathrm{a}}$ change in opposite directions. For example, changing the reflection from $55 \%$ to $60 \%$ would require a decrease in the $\mu_{\mathrm{a}}$ but increase the $\mu_{\mathrm{s}}$ and the converse is true if the reflection was changed from $55 \%$ to $50 \%$.

Given these trends, we hypothesize that the measured peak temperatures were too low. One possible reason is that the thermal camera sampling rate is only $60 \mathrm{~Hz}$ and the camera cannot be triggered with the laser. It was not possible to determine if the thermal camera was recording the peak temperature or some later point as the skin had already started cooled. By employing temperatures that were lower than the true skin surface temperatures, both $\mu_{\mathrm{S}}$ and $\mu_{\mathrm{a}}$ are underestimated for the 1 layer model. If higher $\mu_{\mathrm{s}}\left(\mathrm{derm} \& \mathrm{epi}\right.$ ) and $\mu_{\mathrm{a}}(\mathrm{derm})$ were used in the 2-layer IMC, then the final calculated $\mu_{\mathrm{a}}$ (epi) would be lower for all skin types, thus leading to better validation of the experimental results. Obtaining more accurate peak temperatures should be performed to verify this hypothesis.

This general model has shown promise but refinements are necessary. More accurate methods need to be developed for measuring or calculating temperature.

\section{REFERENCES}

1. Ennow, K., and Jessen, K. A., Spectral measurements and Monte Carlo calculations of scattered radiation from therapeutic radiation sources, Acta Radiol Ther Phys Biol, 14, 262 (1975).

2. Santoro, R. T., Alsmiller, R. G., Jr., and Chandler, K. C., Calculation of the effects caused by bone present in phantoms irradiated by negatively charged pions, Med Phys, 1, 303 (1974).

3. Muthukrishnan, G., and Gopinath, D. V., Charged-particle transport in one-dimensional systems, Radiat Res, 95, 1 (1983).

4. Wilson, B. C., and Adam, G., A Monte Carlo model for the absorption and flux distributions of light in tissue, Med Phys, 10, 824 (1983).

5. Wang, L., Jacques, S. L., and Zheng, L., MCML--Monte Carlo modeling of light transport in multi-layered tissues, Comput Methods Programs Biomed, 47, 131 (1995).

6. Keijzer, M., Jacques, S. L., Prahl, S. A., and Welch, A. J., Light distributions in artery tissue: Monte Carlo simulations for finite-diameter laser beams, Lasers Surg Med, 9, 148 (1989).

7. Marchesini, R., Clemente, C., Pignoli, E., and Brambilla, M., Optical properties of in vitro epidermis and their possible relationship with optical properties of in vivo skin, $J$ Photochem Photobiol B, 16, 127 (1992).

8. Simpson, C. R., Kohl, M., Essenpreis, M., and Cope, M., Near-infrared optical properties of ex vivo human skin and subcutaneous tissues measured using the Monte Carlo inversion technique, Phys Med Biol, 43, 2465 (1998).

9. Star, W. M., Light dosimetry in vivo, Phys Med Biol, 42, 763 (1997).

10. Zeng, H., MacAulay, C., McLean, D. I., and Palcic, B., Reconstruction of in vivo skin autofluorescence spectrum from microscopic properties by Monte Carlo simulation, J Photochem Photobiol B, 38, 234 (1997).

11. Welch, A. J., and van Gemert, M. J. C., Optical-thermal response of laser-irradiated tissue, Plenum Press, New York (1995). 